DOI: https://doi.org/10.29393/EID3-21ETJC70021

\title{
ERGONOMÍA, TRABAJO Y SISTEMA DE SALUD: REFLEXIONES DESDE COLOMBIA
}

\author{
ERGONOMICS, WORK AND HEALTHCARE SYSTEM: REFLEXIONS FROM COLOMBIA
}

\author{
Jairo Ernesto Luna-García* \\ Aida Josefina Rojas-Fajardo** \\ Gabriel García-Acosta ${ }^{* * *}$ \\ Karen Lange-Morales ${ }^{* * * *}$ \\ Juan Carlos Velásquez-Valencia ${ }^{* * * *}$ \\ Lessby Gómez-Salazar ${ }^{* * * * * *}$ \\ Carlos Andrés Fandiño-Losada ${ }^{* * * * * *}$
}

\begin{abstract}
Resumen: Este artículo introduce la línea de investigación "ergonomía, trabajo y salud” propuesta en el proyecto de creación del "doctorado en ergonomía" que adelantan la Universidad del Valle y la Universidad Nacional de Colombia, como parte de la construcción de conocimiento sobre el camino recorrido por la comunidad que ha trabajado la ergonomía en Colombia, en especial desde la implementación de la Ley 100 de 1993, mediante la cual se crea el sistema de seguridad social integral en este país. Este sistema establece tres grandes subsistemas: pensiones, salud y riesgos laborales, siendo este último el principal escenario de aplicación de la ergonomía en Colombia. El artículo reflexiona sobre los retos investigativos que se vislumbran en, para y desde la ergonomía del sistema de salud, en especial ante la actual situación de pandemia. La ergonomía del sistema de salud se entiende desde el compromiso con las instituciones y políticas que configuran este sistema. La ergonomía para el sistema de salud busca abordar las instituciones reales y los procedimientos en el funcionamiento del sistema de salud, entendidos como marcos que regulan la acción humana. La ergonomía en el sistema de salud hace referencia a los estudios, acciones y desarrollos que se adelantan en las instituciones de salud o con relación a los propios trabajadores de la salud. Se concluye que la formación doctoral permitirá a sus egresados transitar de manera interdisciplinar y transdisciplinar, para posicionar la ergonomía en niveles estratégicos y políticos que contribuyan a mejorar los sistemas de salud.
\end{abstract}

\footnotetext{
"Universidad Nacional de Colombia. Bogotá, Colombia. Correo electrónico: jelunag@unal.edu.co. Orcid: https://orcid.org/oooo-0002-0885-7223. Autor de correspondencia.

**Universidad del Valle. Cali, Colombia. Correo electrónico: aida.rojas@correounivalle.edu.co. Orcid: https://orcid.org/oooo-0oo3-2338-9410

${ }^{* * *}$ Universidad Nacional de Colombia. Bogotá, Colombia. Correo electrónico: ggarciaa@unal.edu.co. Orcid: https://orcid.org/oooo-0003-2073-9745

*****Universidad Nacional de Colombia. Bogotá, Colombia. Correo electrónico: klangem@unal.edu.co. Orcid: https://orcid.org/o000-0003-0775-0800

******Universidad del Valle. Cali, Colombia. Correo electrónico: juan.carlos.velasquez@correounivalle.edu.co. Orcid: https://orcid.org/oooo-0003-0139-068o

${ }_{* * * * * *}$ Universidad del Valle. Cali, Colombia. Correo electrónico: lessby.gomez@correounivalle.edu.co. Orcid: https://orcid.org/oooo-0003-2338-9410

********Universidad del Valle. Cali, Colombia. Correo electrónico: carlos.fandino@correounivalle.edu.co. Orcid: https://orcid.org/oooo-0oo2-3385-7380
} 
Palabras clave: Ergonomía, sistemas de salud, Colombia, doctorado en ergonomía.

\begin{abstract}
This article introduces the line of research "ergonomics, work and health" proposed in the project for the creation of the "doctorate in ergonomics" carried out by the Universidad del Valle and the Universidad Nacional de Colombia, as part of the construction of knowledge about the path traveled by the community that has worked on ergonomics in Colombia, especially since the implementation of Law 100 of 1993 , through which the comprehensive social security system is created in this country. This system establishes three large subsystems: pensions, health and occupational risks, the latter being the main application scenario of ergonomics in Colombia. The article reflects on the investigative challenges that are seen in, for and from the ergonomics of the health system, especially in the current pandemic situation. The ergonomics of the health system is understood from the commitment to the institutions and policies that make up this system. Ergonomics for the health system seeks to address the real institutions and procedures in the operation of the health system, understood as frameworks that regulate human action. Ergonomics in the health system refers to the studies, activities, and developments carried out in health institutions or concerning health workers themselves. It is concluded that the doctoral training will allow its graduates to travel in an interdisciplinary and transdisciplinary way, to position ergonomics at strategic and political levels that contribute to improving health systems.
\end{abstract}

Keywords: Ergonomics, health systems, Colombia, doctorate in ergonomics.

Recepción: 02.06.2021 / Revisión: 17.06.2021 / Aceptación: 21.07.2021

\title{
Introducción
}

El punto de partida de la ergonomía estuvo centrado en el estudio de la interacción sujetoobjeto, de manera más precisa en la relación "hombre-máquina”. En diversas vertientes esta relación pasó a ser abordada desde diferentes ámbitos, como la propuesta de Gabriel García Acosta en su texto "La ergonomía desde la visión sistémica" (2002), donde se amplía el análisis al estudio del sistema ergonómico, entendido como el estudio de la interacción entre ser humano y ambiente construido (espacio físico y objetos), con énfasis en el diseño de la interfaz y contemplando la actividad como el alma del sistema y su ubicación en el entorno PESTA (político, económico, social, tecnológico y ambiental).

La definición de ergonomía acogida por la Asociación Internacional de Ergonomía que toma el grupo de trabajo del Doctorado en Ergonomía, proyecto interinstitucional entre la Universidad del Valle y la Universidad Nacional de Colombia, nos permite asumir esta visión sistémica y poder ubicarlo tanto en una dimensión macro como en una visión meso y micro, con lo cual es posible situar el estudio de la relación entre ergonomía y sistema de salud en los siguientes dominios, parafraseando a Margolín (2017), en una dimensión de ergonomía del sistema de salud, para el sistema de salud y en el sistema de salud.

La ergonomía del sistema de salud se entiende en referencia con el compromiso con las instituciones y políticas que configuran este sistema. La pregunta aquí es si desde la ergonomía se pueden recomendar cambios en el sistema de salud y cuál sería la posibilidad de incidir con dichas recomendaciones. La ergonomía para el sistema de salud busca abordar las instituciones reales y los procedimientos en el funcionamiento del sistema de salud, 
entendidos como marcos que regulan la acción humana. Dentro de las instituciones se puede mencionar las Empresas Promotoras de Salud y las Instituciones Prestadoras de Servicios de Salud. Dentro de los procedimientos se contempla tanto el marco normativo para la afiliación como el acceso a los servicios. Aquí se incluye la oportunidad de participación ciudadana en las decisiones, la transparencia y la rendición de cuentas. La ergonomía en el sistema de salud es tal vez el dominio que se aborda con más frecuencia en las investigaciones e intervenciones ergonómicas y hace referencia a los estudios, acciones y desarrollos que se adelantan en las instituciones de salud o con relación a los propios trabajadores de la salud.

De esta forma, se trata de responder a la pregunta: ¿Cómo desde la disciplina se debe proponer un marco conceptual y metodológico propio e integrador aplicable a la comprensión de las relaciones para el diseño y desarrollo de los sistemas de salud?

\section{Materiales y métodos}

La Universidad del Valle y la Universidad Nacional de Colombia adelantan la creación de un Doctorado Conjunto en Ergonomía, programa que busca consolidar los estudios ergonómicos de nuestros países latinoamericanos, tanto en materia de investigación científica (teorías y abordajes metodológicos) como en el ejercicio profesional (Velásquez-Valencia et al., 2021).

Algunos de los casos prioritarios que motivan este proyecto son las condiciones de trabajo y empleo que requieren de investigaciones integrales, multi y transdisciplinares, en las que se contemple el mejoramiento de los ambientes socioculturales, económicos y educativos, para la salud y el bienestar de los trabajadores. Además, es fundamental reflexionar y mejorar las condiciones en las que se ha realizado la transferencia de tecnología y la innovación en los países considerados de la 'periferia', cuya implementación muchas veces provoca sinsabores, debido a la falta de una correcta concepción situada y adaptación causada por incompatibilidades físicas y cognitivas y el olvido de la idiosincrasia local. Una apropiada transferencia tecnológica requiere tener en cuenta todos los factores que entran en juego: desde los físicos, tales como los estándares antropométricos, hasta las condiciones más intangibles como la cultura. El doctorado se quiere orientar en la línea de estimular los procesos de innovación tecnológica, para fortalecer a los procesos de transición y autonomía. Por otra parte, en América Latina, impulsada por la denominada economía informal, la ergonomía debe permitir comprender los «asuntos de interés» (Matters of Concern), como lo formula Bruno Latour (Stephan, 2015) y valorar tanto lo positivo, como lo que enseña la economía popular frente a las condiciones de trabajo y vida.

Hay numerosos campos por trabajar como un imperativo ético de la ergonomía. La investigación científica y el aporte a las soluciones de problemáticas en los campos de la ergonomía requieren importantes reflexiones prospectivas sobre el futuro del trabajo y el trabajo del futuro, el trabajo asociado a la economía popular, el envejecimiento y su relación con las poblaciones vulnerables, las nuevas tecnologías y los desarrollos técnico-científicos, entre otros.

Dichos retos podrán asumirse bajo la concepción del pensamiento sistémico - complejo y desde la ecología de saberes, para los diferentes campos de la actividad humana (de Sousa- 
Santos, 2009). Considerando la pandemia que se está viviendo, es de resaltar la importancia de la aplicación de la ergonomía al tele-trabajo y al trabajo a distancia, a los procesos de enseñanza-aprendizaje mediados por la virtualidad y a las nuevas propuestas pedagógicas que demanda la actual situación (de Sousa-Santos, 2020). Hoy es necesario pensar futuros distintos, confrontando las ideas de progreso y desarrollo que están en el centro de la crisis y la búsqueda de alternativas (Klein, 2015).

En el marco de este proyecto educativo, se definieron tres líneas de investigación. Una de ellas, Ergonomía, Trabajo y Salud, que sirve de marco de reflexión para el enfoque de este artículo, orientado hacia los sistemas de salud como uno de los escenarios de esta línea de trabajo.

La ergonomía se concibe y desarrolla históricamente a partir de los procesos laborales, inicialmente tiene como objeto de su intervención la relación entre los humanos y las máquinas para posteriormente involucrar la relación de éstos (humano - máquina) con el medio ambiente.

El propósito de la ergonomía en los ambientes laborales se centra en preservar la salud, el bienestar de los humanos, la prevención de las enfermedades y los accidentes laborales, mejorar el desempeño humano y además los efectos que este pueda tener sobre los sistemas productivos, procurando también un incremento en la productividad y calidad de los productos y servicios.

Es así como el campo de acción de la ergonomía en el trabajo comprende todas las esferas del desempeño humano, tanto individual como colectivos; no solo estudia las relaciones más inmediatas con las tareas o con el puesto de trabajo, sino que también lo hace con el proceso en general, es decir cómo se organizan y dividen los procesos laborales, cómo interactúan los individuos con los equipos, entorno y especialmente entre los seres humanos.

Esta visión de la ergonomía del trabajo se centra por lo tanto en la protección del trabajador, en su confort y bienestar, así como en el cuidado e incremento de la productividad, eficiencia de los procesos, desempeño y diseño de los sistemas productivos en su conjunto.

Bajo esta óptica son múltiples los problemas que se plantea resolver la ergonomía en los ámbitos laborales; una de las grandes preocupaciones ha sido la prevención de las enfermedades especialmente los desórdenes musculoesqueléticos, para lo cual ha desarrollado diversos métodos de diagnósticos de los factores de riesgo que se asocian con estos trastornos y mecanismos de diseño e intervención de los ambientes y procesos laborales para su prevención y control.

También ha sido el interés de la ergonomía estudiar y transformar los aspectos concernientes al aprendizaje, la memoria, la atención, es decir el desempeño cognitivo de los trabajadores y los factores psico-laborales, así como la relación existente entre la organización del trabajo, la jornada laboral, los turnos, etcétera, con el fin de comprender mejor estas relaciones para preservar el bienestar humano en el trabajo y el óptimo desempeño del sistema.

A su vez la ergonomía del trabajo ha ampliado su campo de estudio a aspectos 
concernientes a la comprensión del desempeño humano individual y colectivo, a la forma como se comunican y establecen relaciones los grupos laborales, las formas de dirección, las distintas expresiones de poder y la interrelación entre los sistemas sociotécnicos.

Esta visión de la ergonomía, como podemos observar, se centra en los procesos productivos, es allí donde se establece su quehacer como disciplina y profesión. Sin embargo, existe otra tendencia que centra su acción en los trabajadores no sólo como fuerza de trabajo, es decir, ligado a la producción de bienes y servicios cuyo objetivo, como se explicó es la prevención de enfermedades y accidentes, sino que también se preocupa por comprender cómo lo afectan los procesos de consumo.

Esta visión de la ergonomía se extiende al trabajador en toda su dimensión, trasciende el ámbito laboral y amplía su intervención a los escenarios no laborales donde el trabajador como individuo y colectivo desarrolla otro tipo de actividades (procesos de consumo) como el descanso, la recreación, la educación, entre otras.

La ergonomía reconoce por lo tanto en estos ambientes de trabajo y de consumo, no solo la identificación y control de condiciones que pueden deteriorar la salud sino, $\mathrm{y}$ especialmente, los determinantes y la determinación de los procesos de salud y enfermedad y cómo estos afectan su desempeño social.

Bajo esta dinámica la ergonomía amplía su marco de acción y recoge y nutre su accionar, permeado por la cultura, la política, las normas y leyes, y demás fenómenos que determinan la actividad humana y los efectos de la misma en los procesos productivos y de consumo es decir en los procesos sociales, entendiendo que es el trabajador el núcleo central de esta relación.

Por lo tanto, la ergonomía desde el enfoque de la salud de los trabajadores amplía su marco de acción no solo a la protección en los escenarios laborales, sino que se interesa por la protección integral del trabajador, del diseño de los ámbitos donde vive y trabaja; bajo esta perspectiva las relaciones sociales y todo lo que las afectan pasan a ser el objeto de estudio de la ergonomía, pretendiendo con ello el bienestar del trabajador y el buen vivir con el entorno.

En relación con lo anterior, se puede concluir que algunos de los temas de interés de la línea de investigación ergonomía, trabajo y salud son: Análisis de la accidentalidad laboral, desde una perspectiva de complejidad; estudio de las situaciones de trabajo y la prevención de los desórdenes musculoesqueléticos; integridad mental, ergología y prevención de riesgos psicosociales; las condiciones de trabajo en la economía informal; la seguridad del paciente y el cuidador, y la gestión y organización de los servicios de salud.

En este sentido, algunas de las investigaciones adelantadas directamente por los investigadores involucrados en proyecto doctoral incluyen el síndrome de Burnout en médicos iberoamericanos (Cobo-Molina et al., 2019), el estudio de las condiciones laborales psicosociales del personal de la Secretaría Distrital de Salud de Bogotá (Rubio-Castro \& Luna-García, 2017), la gestión de la motivación laboral en el personal de enfermería (TriviñoCervera et al., 2017) y la violencia en el trabajo del sector público de la salud (Luna-García et al., 2015), entre otros.

Desde estos desarrollos a continuación se profundiza en la perspectiva frente a las 
posibilidades de la ergonomía del, para y en el sistema de salud.

\section{Resultados y discusión}

\section{Ergonomía del sistema de salud}

En Colombia, los aportes desde las áreas de la ergonomía al sistema de salud han sido mínimos. La Ley 1562 de 2012 del Congreso de la República de Colombia, la cual es la modificación más reciente sobre el Sistema de Riesgos Laborales de Colombia, no menciona los términos "ergonomía", "factores ergonómicos" o "factores humanos", enfocándose solamente en los aspectos más tradicionales de seguridad e higiene industrial, sin tener en cuenta el abordaje sistémico, holístico y transdisciplinar que aporta la ergonomía (LunaGarcía, 2014). En contraste, en el Decreto 1443 del Sistema de Gestión de Seguridad y Salud (Ministerio del Trabajo de la República de Colombia, 2014) sí se menciona los factores ergonómicos o biomecánicos como parte de las condiciones y medio ambiente de trabajo, y se mencionan los peligros ergonómicos como parte de las actividades de identificación de peligros, evaluación y valoración de los riesgos laborales. Adicionalmente, revisiones documentales recientes sobre la investigación en ergonomía en Colombia identifican las áreas de aplicación física y cognitiva como las temáticas que se abordaron con mayor frecuencia en las investigaciones realizadas (Silva, 2011; Andrade-Jaramillo \& CarolinaGómez, 2013; García-Acosta et al. 2008). Por otro lado, las escasas investigaciones en el tema de la ergonomía organizacional apuntan a aspectos específicos de las empresas u otros entornos laborales, sin enfocarse en la organización del Sistema General de Seguridad Social de Colombia (Silva, 2011; Andrade-Jaramillo \& Carolina-Gómez, 2013; García-Acosta et al. 2008).

La visión sistémica de la ergonomía permite abordar el problema de la salud desde diferentes esferas. La primera está centrada en la comprensión de los servicios de salud desde la atención primaria en salud hasta los niveles de mayor complejidad; en esta se articulan los aspectos, científico técnicos y administrativos para el diseño, organización, desarrollo y prestación de los servicios de salud. Su principal aporte radica en la comprensión de las relaciones entre los usuarios de los servicios de salud y la estructura y funcionamiento de los servicios de salud, donde se incluyen los subsistemas socio técnicos y el talento humano en salud, por un lado, y los factores del entorno o contexto, por el otro. Por ejemplo, la ergonomía se aplica desde el diseño de una herramienta o equipo (una prótesis para rehabilitación) hasta el diseño de estándares o guías para la promoción de la salud, prevención o atención especializada. Éstos, a su vez, se convierten en la segunda esfera donde la ergonomía centra su accionar, en la comprensión del contexto donde se desarrollan las actividades de los servicios de salud, es decir los aspectos culturales, ambientales, económicos, jurídicos y políticos que determinan el accionar de la ergonomía en el sistema de salud.

Sin embargo, la ergonomía no centra su accionar sólo en la comprensión de estas relaciones de las actividades humanas con los entornos laborales, sino que principalmente se centra en el diseño y transformación de las mismas; de allí que un tercer nivel de 
intervención de la ergonomía está en el diseño de normas y políticas aplicables a los diversos ámbitos en los que se desarrolla el proceso de la salud y de la atención de la salud. A estos ámbitos los podríamos llamar microsistemas locales o regionales, de allí la importancia del análisis de la actividad humana. Un cuarto ámbito, más general que incluye a los anteriores, está en comprender cómo se articulan en los diferentes niveles estos microsistemas de salud en un sistema integral de salud, por lo tanto, la ergonomía está llamada a brindar soporte conceptual y metodológico para el diseño, desarrollo y transformación de los sistemas de salud. Para ello se sirve de su enfoque sistémico, basado en diversas metodologías propias del desarrollo de la ergonomía y de las relaciones que ésta pueda hacer con otras disciplinas, es decir, la ergonomía debe cumplir un papel integrador necesario y fundamental en la construcción de las políticas y desarrollo del macrosistema de salud.

Desde la visión de la Asociación Internacional de Ergonomía (IEA, por sus siglas en inglés), la ergonomía contribuye al diseño y desarrollo de sistemas seguros y sostenibles mediante tres componentes de la intervención ergonómica:

- Adopta un enfoque de sistemas, utilizando un proceso sistemático paso a paso, mediante un abordaje holístico.

- Se basa en el diseño, en este caso apoyándose en metodologías como el diseño universal o el diseño inclusivo y el diseño participativo.

- Se centra en optimizar los resultados que son fundamentales para cualquier sistema, especialmente los sistemas de salud: el desempeño del sistema y el bienestar humano, los cuales están íntimamente ligados.

A su vez considera un enfoque participativo, partiendo del principio de que cualquier persona o comunidad que pueda verse afectada por una decisión o actividad de la intervención ergonómica es una parte interesada. Entonces, los actores se pueden agrupar como:

- Los influenciadores del sistema, como por ejemplo las autoridades competentes, ministros, secretarios de salud, entes reguladores, organismos normalizadores, sociedades científicas, etc.

- Responsables del sistema, y aquellos que toman decisiones sobre los requisitos para el desarrollo del sistema, en los ámbitos de la promoción, la prevención y la atención en salud.

- Los expertos del sistema, el talento humano en salud en los diversos escenarios de acción desde el plano administrativo hasta el asistencial más especializado.

- Las personas, las comunidades y sus territorios, que forman parte esencial del sistema, son los usuarios y las acciones del sistema los afecta directamente.

Desde esta perspectiva, las acciones de la ergonomía deben integrarse en diversos niveles desde el internacional al local, participando en los organismos internacionales para la construcción de las recomendaciones para mejorar el bienestar de las personas beneficiadas por el sistema de salud y que laboran en el sistema de salud. De esta forma, los niveles de acción son los siguientes: 
- En el ámbito nacional para la construcción de la política pública y las normas generales del sistema de salud.

- En el plano educativo para la formación del talento humano en salud y la educación de los actores del sistema.

- En el ámbito del trabajo para la formulación de las políticas públicas y la normatividad necesaria para promover, mantener y recuperar la salud de los trabajadores; con especial énfasis en los trabajadores de los sectores informales de la economía.

- Y en el nivel de la praxis: directores ejecutivos y gerentes en empresas, diseñadores de sistemas de atención y del sistema de trabajo, los profesionales en dominios relevantes y los usuarios.

Bajo estos principios, se debe articular la intervención ergonómica entendida no como un molde o modelo predeterminado, sino como un proceso de construcción permanente que debe considerar cada realidad con las particularidades y relaciones contextuales que la determinan.

\section{Ergonomía para el sistema de salud}

A partir de la Ley 100 de 1993 y otras normas posteriores, en Colombia se creó el Sistema General de Seguridad Social en Salud (SGSSS), el cual incluye el conjunto de instituciones, normas y procedimientos mediante los cuales el estado colombiano busca garantizar la prestación de servicios de salud a la población del país (Congreso de la República de Colombia, 1993). Este gran sistema está dividido en tres subsistemas: salud, pensiones y riesgos laborales. Cada uno de estos subsistemas está dirigido a grupos poblacionales específicos. El subsistema de salud, en principio, cobija a todos los habitantes de este país. El subsistema de pensiones abarca a todas las personas con capacidad de aportar a dicho sistema, siendo en su mayoría aquellos que tienen contratos de trabajo, aunque también quienes trabajan de manera independiente tienen la posibilidad de aportar. Por último, el subsistema de riesgos laborales cubre a las personas que trabajan de manera formalizada, aunque también existe la posibilidad de que quienes trabajan de manera autónoma puedan afiliarse. En este contexto, entendemos la ergonomía para el sistema de salud como las instituciones y los procedimientos que regulan la acción humana en los procesos de salud enfermedad, dentro de la estructura legal establecida en Colombia.

Las acciones de la ergonomía se han concentrado en el subsistema de riesgos laborales y, a su vez, como un pequeño apartado, dentro del sistema de gestión de la salud y seguridad en el trabajo (SGSST). En este sentido, en el subsistema de salud, la ergonomía aparece marginalmente en las instituciones y procedimientos, atendiendo a la población que labora en dichas instituciones, quedando por fuera de la lógica del SGSST, tanto las organizaciones en sí como los pacientes y sus familias.

Estructuralmente, el subsistema de salud se compone de dos grandes elementos: las entidades prestadoras de salud (EPS) y las instituciones prestadoras de servicios de salud (IPS). Las EPS, a su vez, se dividen en dos grupos: el régimen contributivo y el régimen subsidiado. Las EPS del régimen contributivo son las encargadas de la afiliación y recaudo de los aportes de empleadores y trabajadores, mientras que las EPS del régimen subsidiado 
(EPS-S) se responsabilizan de afiliar a la población pobre y vulnerable, como una vía de acceso al ejercicio del derecho fundamental de la salud. En otras palabras, las EPS son los encargados de remitir a la gente para la atención a las IPS, teniendo únicamente la labor de intermediación entre el paciente y los hospitales, clínicas y demás IPS. Por su parte, las IPS son quienes prestan los servicios, atendiendo a pacientes de diferentes EPS, y luego facturando los servicios para que sean pagados por la EPS.

Si bien la organización actual del sistema de salud en empresas administradoras y empresas prestadoras podría parecer eficiente en términos de cobertura, las prácticas diarias han mostrado grandes problemas que podrían analizarse y resolverse desde los diferentes dominios de la ergonomía, en especial desde la ergonomía organizacional. En este sentido, se harán algunas reflexiones, desde las posibilidades de la intervención de la ergonomía, sobre algunos aspectos de tres procedimientos: la afiliación al sistema, el acceso a la atención en el sistema y el circuito de cobro y pago de servicios entre las EPS y las IPS.

El proceso de afiliación / desafiliación al sistema presenta grandes retos. Para los trabajadores formales, los formatos y documentos para la afiliación son gestionados por las empresas. Ahora bien, para los trabajadores independientes, el proceso de afiliación debe ser gestionado por ellos mismos, lo que genera una demanda cognitiva y la posibilidad de cometer errores que se puede traducir en que quede por fuera del sistema sin posibilidad de atención o en sanciones. Esta situación ha llevado a que muchas personas no se afilien o se pierda la regularidad de su afiliación, con las consecuencias en su historial médico y la continuidad en tratamiento, entre otros.

Una vez se tiene acceso a la atención en el sistema, la práctica ha mostrado grandes deficiencias que van desde el limitado tiempo de atención de las citas (15 minutos para general y especializadas) hasta el tiempo de espera para tener una cita o un servicio especializado (más de un mes). Este tema resulta especialmente importante en el caso de terapias físicas y psiquiátricas, por poner solo unos ejemplos. ¿Cómo se rehabilita una mano con 10 sesiones de terapia una vez a la semana? O ¿cómo se recupera una persona de un episodio psicótico con una cita de 20 minutos cada tres meses? Esto lleva a que, quienes pueden pagarlo, busquen alternativas fuera del sistema. De acuerdo con Cedetrabajo, recientemente más de 7.5 millones de personas utilizaron recursos propios para la atención a su núcleo familiar (LD Jiménez Molinello, 6 de mayo de 2021).

Como se dijo anteriormente, las EPS y las IPS son las estructuras básicas del sistema, lo cual implica una interrelación fundamental. En esta relación, uno de los circuitos más importantes es lo que en las IPS se denomina "ciclo de ingreso". Cada procedimiento, dispositivos médicos y medicinas son facturadas, lo cual requiere de una meticulosa elaboración de documentos por parte de la IPS. Las EPS reciben estas facturas y las analizan, colocando frecuentemente glosas para demorar el pago o no pagar dichas cuentas.

¿Qué puede hacer la ergonomía para el sistema de salud? Vemos posibilidad de intervención en varias escalas. Un primer nivel está en la simplificación de procesos y gestiones. Dos ejemplos de trabajos en esta escala se realizaron en el Hospital Universitario Nacional entre el 2018 y el 2020, con la participación de estudiantes de Ergonomía Organizacional analizando el Ciclo de Ingreso de la institución, así como con una 
investigación de Maestría en Ingeniería Industrial (Niño-Sánchez, 2020). Otra posibilidad de intervención en esta escala está en el mejoramiento de la usabilidad de los sistemas informáticos, componentes clave del sistema. En línea con lo anterior se pueden abordar proyectos como los desarrollados en Vivelab para reducir la brecha digital, denominados como vive digital (BGA, 2018).

Una segunda oportunidad está en la aplicación de los métodos de la ergonomía misma, en especial la ergonomía participativa, para propiciar la comprensión o el rediseño del sistema mismo entre las diferentes partes interesadas. Una investigación que explora esta posibilidad versa sobre el uso de dispositivos médicos en sala de operaciones, presentando una comprensión de las dinámicas que se dan en las intervenciones quirúrgicas en traumatología, desde una visión sistémica y como prácticas sociomateriales (Lange-Morales, 2017).

Una tercera escala de intervención está en la comprensión del sistema de salud desde los dos pilares del propósito de la ergonomía i.e. la búsqueda de bienestar y eficiencia de manera paralela y complementaria, contribuyendo así a superar la visión economicista que domina el actual modelo. Cuando las personas, en especial quienes deciden e inciden en el diseño de los sistemas, entienden que el bienestar no está en contraposición de la eficiencia, se potencian los resultados positivos y la transformación estratégica.

Finalmente, la ergonomía para el sistema de salud debería desplegarse en los niveles táctico, estratégico y político, pues su actual implementación se hace de manera coyuntural y desarticulada de las políticas de innovación y transformación o cambio tecnológico. La intervención en los puestos de trabajo se reduce a recomendaciones o acciones en el nivel operacional.

"En la actualidad no se ha dado la relevancia suficiente ni la divulgación necesaria dentro de las organizaciones productivas al papel del ergónomo y de la ergonomía en los procesos de innovación. Además, los ergónomos aún no se ven con el rol de ser parte de un equipo de innovación. La acción ergonómica debe dejar de ser asociada y limitada al ámbito de las aseguradoras de riesgos laborales" (García-Acosta \& Lange-Morales, 2011, p 47).

\section{Ergonomía en el sistema de salud}

Uno de los objetivos de la ergonomía es lograr una relación lógica y apropiada entre el individuo y elementos del sistema, a partir del análisis no solo de la seguridad física, los aspectos cognitivos y psicosociales de la vida y el trabajo, sino también de los aspectos relacionados con la interacción del hombre con las herramientas, su diseño y aquellos factores relacionados con la organización, el tipo del trabajo y las interacciones que se generan en el desarrollo de una actividad laboral (IEA). Bajo esta mirada, como se apreciará a continuación, se enmarcan la mayoría de las investigaciones realizadas en Colombia, tanto en profesionales de la Salud como en las propias Instituciones Prestadoras de servicios de Salud.

Al realizar un rastreo sobre trabajos cuyo dominio es la ergonomía en relación con esfuerzo biomecánico en la movilización de pacientes, trastornos musculoesqueléticos, condiciones de trabajo psicosociales y la carga mental en Colombia, se identificaron 56 
artículos publicados en español en los últimos 5 años, que cumplen con dichas categorías y sobre los cuales se pudo tener acceso al texto completo.

Dichos artículos fueron clasificados de acuerdo con los dominios de especialización propuestos por la IEA en el año 2000: Ergonomía Física, Ergonomía Cognitiva y Ergonomía Organizacional. Como parte del análisis se encontraron algunos estudios que no se ubicaban dentro del marco de los dominios expuestos anteriormente, esto porque tratan temáticas que se relacionan con el tema de ergonomía, pero no se centran en ella o tienen que ver con su aplicación. Por esto, dichos trabajos, se categorizaron como otros o aplicada.

El mayor número de artículos analizados para este estudio se encuentra en el dominio de especialización de ergonomía cognitiva en donde se nomina como riesgo psicosocial, estrés, síndrome de Burnout, sobre carga laboral, carga mental, salud mental, síndromes depresivos, calidad de vida-salud mental y fatiga. Dentro de esta subcategoría se hace evidente que la nominación y por tanto el tema más utilizado es riesgo psicosocial. A manera de ejemplo, las autoras Amado, Mayorga y Núñez (2021), en su trabajo de grado en la especialización en gerencia en seguridad y salud en el trabajo, abordan la temática, específicamente en el personal de fisioterapia que se encuentra en centros de salud de atención ambulatoria y centros de salud de Colombia, en donde buscan identificar cómo este aspecto los afecta su calidad de vida y su desempeño dentro de sus puestos de trabajo. Así mismo Galindo y Joya (2017), investigan sobre las diferentes condiciones de trabajo del personal de auxiliar de enfermería y su incidencia frente al riesgo psicosocial en un hospital de la red pública de la ciudad de Bogotá. De igual manera las otras investigaciones, lo relacionan con puestos de trabajo de médicos, enfermeras y personal en general de instituciones psiquiátricas. Todos estos artículos, evidencian el alto riesgo psicosocial en esta población, derivada de las acciones propias de los profesionales, pero también con condiciones laborales insatisfechas que generan una alta demanda física y mental en esta población.

Frente al dominio de especialización de ergonomía física, se encuentran que hay nominaciones con relación a desórdenes músculo - esqueléticos, fatiga física, lesiones osteomusculares, lumbalgia ocupacional y postura. Por ejemplo, según Velasco-Rey (2016), las posturas que se deben adoptar por parte de los instrumentadores quirúrgicos pueden afectar el sistema musculoesquelético. En este aspecto inciden diferentes factores como, si son posturas estáticas o dinámicas, el tipo de cirugía, la ubicación de las mesas de instrumental, la altura de la cama de cirugía, características personales y algunas condiciones técnicas del procedimiento a asistir. Este artículo, por ejemplo, deja ver como se relacionan diferentes aspectos con el tema músculo-esquelético. Aspecto que es relevante y que es reiterativo en la mayoría de los trabajos revisados.

$\mathrm{Al}$ hacer referencia a la ergonomía organizacional, se evidencian temas relacionados con sistema de seguridad y salud en el trabajo, condiciones laborales, factores de riesgo que llevan a accidentes laborales, condiciones de salud y trabajo, condiciones de trabajo, empleo y precariedad. Las áreas de trabajo investigadas dentro de estas temáticas son funcionarios contratistas del área de la salud, enfermería, áreas quirúrgicas, área de urgencias y unidad de cuidados intensivos. Entre otros trabajos, se encuentra el de Burgos (2018), quien detecta que el rendimiento en los equipos de trabajo es deficiente, con relación a la obtención de 
objetivos, partiendo de la motivación de los equipos de trabajo en las instituciones del sector Salud de Colombia. Lo cual indica que la motivación es un impulso importante para el rendimiento de los equipos.

Ahora bien, frente a la ergonomía aplicada y los otros trabajos se encuentra que hay propuestas de diseños de instrumentos de valoración ergonómica, diseño y aplicación de un modelo de medición de satisfacción laboral para teletrabajadores, diseño de un aditamento para instrumental quirúrgico y revisión bibliográfica. Wilcken (2019) presenta en su trabajo de grado "Diseño y manufactura de un mango mejorado para instrumental de cirugía laparoscópica, haciendo énfasis en la ergonomía y la personalización del instrumental”, con este trabajo se busca rediseñar la ergonomía de los mangos de instrumentos existentes para lograr una mayor comodidad del médico que hace la intervención.

En cuanto a los resultados en el dominio de ergonomía cognitiva, llama la atención que ha aumentado la cantidad de investigaciones en el área, de acuerdo con un estudio realizado por Edwin Giovanni Silva en el 2010, en donde el dominio de esta área era la más baja. Este fenómeno puede estarse dando porque durante los últimos años el tema cognitivo ha tomado gran relevancia, por la incidencia directa que tiene frente al desempeño de los trabajadores.

Estos resultados, evidencian una tendencia a considerar la aplicabilidad de la ergonomía en instituciones de salud, hacia la detección, valoración y control del riesgo laboral, por lo que es necesario mayores desarrollos en campos interdisciplinares, bajo la mirada de la ergonomía como el estudio de sistemas de comportamiento complejo (GarcíaAcosta, 2002), que en este caso, comprende el estudio de las redes de interacción con otras instituciones, pacientes, familias y la propia sociedad, que a su vez que interactúan de manera no lineal en diferentes escalas que generan consecuencias a veces no deseadas como reacciones adversas a medicamentos, infecciones nosocomiales, reingresos hospitalarios y deterioro funcional (Lipsitz, 2012).

Bajo este enfoque, las intervenciones ergonómicas deben también abarcar campos como la seguridad del paciente, el análisis de la eficacia del personal, la reducción de los errores y el aumento de la satisfacción del personal y los usuarios, todo ello orientado a mejorar la calidad de la asistencia sanitaria. Con relación al entorno, aspectos como el diseño de edificios, el mobiliario y equipos médicos son puntos de profundización en la investigación en ergonomía y sobre lo cual existen menos publicaciones. Así mismo, desde la Ergoecología que se ocupa del estudio del ser humano y sus relaciones con el ambiente para establecer, analizar, mitigar, prevenir, controlar y corregir sus impactos (Saravia-Pinilla, 2005), es necesario considerar la forma en que estas instituciones afectan el medio ambiente en temas como el manejo de residuos biológicos y sanitarios.

En el marco del Doctorado en Ergonomía, se ha considerado además la importancia de avanzar en una ergonomía enfocada a las necesidades y condiciones de Latinoamérica y otros países del Sur Global. En este caso, es necesario considerar la existencia de un espacio infranqueable entre las condiciones ergonómicas de las instituciones de salud públicas y privadas, que reflejan inequidades sociales, que afectan no solo la vida de los pacientes sino también del personal que en ellas laboran. Ello supone, la importancia de examinar como elemento del sistema ergonómico, las condiciones políticas y económicas de la región y el 
país, así como la de cada institución de salud, como factores que facilitan u obstaculizan la implementación de acciones ergonómicas apropiadas.

\section{Conclusiones}

Unas consideraciones finales permiten presentar retos emergentes derivados de la pandemia por Covid-19 a manera de conclusiones.

Es importante resaltar que la formación doctoral en ergonomía propuesta por la Universidad del Valle y la Universidad Nacional, permitirá a los egresados transitar de manera interdisciplinar y transdisciplinar, para posicionar la ergonomía en niveles estratégicos y políticos que mejoren los sistemas de salud.

El perfil doctoral del egresado le permitirá actuar sin quedar reducidos a un simple factor en el SGSST, y mucho menos relegados a acciones coyunturales, desarticuladas y diagnósticas, sino más bien estratégicas y políticas, sumándose para el caso de los sistemas de salud a los enfoques del campo problemático de la Salud Pública.

La pandemia por Covid-19 emergió como una experiencia crítica que involucró al conjunto de la humanidad en un corto lapso, con un importante impacto en el mundo del trabajo, poniendo de presente nuestra vulnerabilidad y la estrecha dependencia del mundo natural (Luna-García \& Torres-Tovar, 2020). Una de las dimensiones relevantes de la crisis es la que tiene que ver con la importancia de los sistemas de salud, evidenciando la capacidad de respuesta disímil para garantizar el derecho a la salud, desde aquellos centrados en el aseguramiento universal frente a los que propenden por un acceso universal (Hernández et al., 2021).

Para la ergonomía resulta un reto contribuir en la construcción de respuestas en la adecuación de los sistemas de salud para atender de mejor manera los estrictos requerimientos de bioseguridad, pero también ayudar a la reconfiguración de los servicios que permitan el cabal cumplimiento de las medidas de control no farmacológicas, tanto en su dimensión de distanciamiento físico, mitigación del impacto psicosocial, pero también en una dimensión organizacional, que contribuyan a la protección de trabajadores y pacientes, en un marco de respeto y promoción de los derechos humanos. En este horizonte es que planteamos la propuesta de doctorado en ergonomía. 


\section{Referencias}

Amado, I. Mayorga, E., \& Núñez, A. (2021). Análisis de los factores de riesgo psicosocial en terapeutas físicos en los centros sanitarios de atención ambulatoria y hospitalaria en Colombia [tesis de especialización, Universidad ECCI]. Repositorio Digital ECCI. https://repositorio.ecci.edu.co/handle/001/895

Andrade-Jaramillo, V., \& Carolina-Gómez, I. (2013). Salud laboral investigaciones realizadas en

Pensamiento

Psicológico,

4(10).

https://revistas.javerianacali.edu.co/index.php/pensamientopsicologico/article/view/701

BGA (2018). Regresa el servicio gratuito a los 8 puntos Vive Digital de Bucaramanga. Alcaldía de Bucaramanga. Prensa BGA. https://www.bucaramanga.gov.co/noticias/regresa-el-serviciogratuito-a-los-8-puntos-vive-digital-de-bucaramanga/

Burgos, M. (2018). Motivación y rendimiento laboral en instituciones del sector salud de Colombia. Consensus (Santiago). Revista Interdisciplinaria de Investigación, 2(2), 21-41. https://pragmatika.cl/review/index.php/consensus/article/view/20

Cobo-Molina, N., Álvarez-Mena, J., Parra-Osorio, L., Gómez-Salazar, L., \& Acosta-Fernández, M. (2019). Prevalencia del síndrome de Burnout en médicos iberoamericanos entre 2012 y 2018: una revisión sistemática. Diálogos de Saberes, (50), 39-6o. https://doi.org/10.18041/0124o021/dialogos.50.2019.5551

Congreso de la República de Colombia (1993). Ley número 100 de 1993 por la cual se crea el Sistema de Seguridad Social Integral $y$ se dictan otras disposiciones. https://www.minsalud.gov.co/sites/rid/Lists/BibliotecaDigital/RIDE/DE/DIJ/ley-100-de1993.pdf

de Souza-Santos, B. (2009). Una epistemología del sur. La reinvención del conocimiento y la emancipación social. México: Consejo Latinoamericano de Ciencias Sociales - Siglo XXI Editores.

de Sousa-Santos, B. (2020). La cruel pedagogía del virus. Buenos Aires: CLACSO. http://biblioteca.clacso.edu.ar/clacso/se/20200430083046/La-cruel-pedagogia-delvirus.pdf

Galindo, W., \& Joya, C. (2017). Factores de riesgos psicosociales en auxiliares de enfermería de un hospital de la red pública en la ciudad de Bogotá, Colombia. Revista U.D.C.A Actualidad \& Divulgación Científica, $\quad 20(1), \quad 23-32$. http://www.scielo.org.co/pdf/rudca/v20n1/v2on1ao4.pdf

García-Acosta, G. (2002). La ergonomía desde la visión sistémica. Bogotá: Unibiblos.

García-Acosta, G. \& Lange-Morales, K. (2011) La ergonomía como estructura de innovación en la ingeniería de proyectos de organizaciones productivas. Bogotá: Universidad Nacional de Colombia. https://www.uneditorial.com/media/hipertexto/carpeta 6/9789587752052.pdf

García-Acosta, G., Lange-Morales, K., Baquero-Yordan, M., \& Ramos-Mora, M. P. (2008). Diez años de ergonomía en el Banco de la República: De la mano en pro de la salud y la productividad. VI Congreso Internacional de Prevención de Riesgos Laborales, ORP 2008. A Coruña, España.

Hernández-Álvarez, M., Henao-Kaffure, L., Eslava-Castañeda, J. C., Vega-Vargas, M., ArrublaSánchez, D., Rodríguez-Ruiz, J. \& Vega-Vargas, M. (2021). La pandemia de la enfermedad por coronavirus (Covid-19) desde una perspectiva crítica histórico-territorial. En C. Tetelboin-Henrion, D. Iturrieta-Henríquez \& C. Schor-Landman (coords.), América Latina: sociedad, política y salud en tiempos de pandemias (pp. 71-114). CLACSO, Universidad 
Autónoma Metropolitana, Universidad Veracruzana, Universidad Mayor de San Simón, Universidad de Valparaíso.

Klein, N. (2015). Esto lo cambia todo: El capitalismo contra el clima. España: Paidos Iberoamérica.

Lange-Morales, K. (2017). Compatibilidades en las multiplicidades: El uso de dispositivos médicos en la sala de operaciones [tesis doctoral, Universidad Nacional de Colombia]. Repositorio Institucional UN. https://repositorio.unal.edu.co/handle/unal/59321

Lipsitz, L. A. (2012). Understanding health care as a complex system: The foundation for unintended consequences. Journal of the American Medical Association, 308(3), 243-244. https://doi.org/10.1001/jama.2012.7551

Luna-García, J. E. (2014). La ergonomía en la construcción de la salud de los trabajadores en $\begin{array}{llllll}\text { Colombia. Revista } & \text { Ciencias de la Salud, } & \text { 12, } & \text { 77-82. }\end{array}$ http://www.scielo.org.co/pdf/recis/v12s1/v12s1a08.pdf

Luna-García, J. E, Urrego-Mendoza, Z., Gutiérrez-Robayo, M., \& Martínez-Durán, A. (2015). Violencia en el trabajo del sector público de la salud: Una visión desde las personas trabajadoras. Bogotá, Colombia. 2011-2012. Revista de la Facultad de Medicina, 63(3), 407417. http://dx.doi.org/10.15446/revfacmed.v63n3.51027

Luna-García, J. E. \& Torres-Tovar, M. (2020). 2020: Pandemia por Covid-19 y mundo del trabajo: Una mirada desde los sectores subalternos. Laboreal, 16(2). http://journals.openedition.org/laboreal/17427

Margolín, V. (2017). Construir un mundo mejor: Diseño y responsabilidad social. México: Editorial Designio.

Ministerio del Trabajo de la República de Colombia (2014). Decreto número 1443 de 2014 por el cual se dictan disposiciones para la implementación del Sistema de Gestión de la Seguridad y Salud en el Trabajo https://www.mintrabajo.gov.co/documents/20147/36482/decreto 1443 sgsss.pdf/ac41ab7 o-e369-9990-c6f4-1774e8d9a5fa

Niño-Sánchez, L. F. (2020). Transformación organizacional en el Hospital Universitario Nacional de Colombia (HUN). Una propuesta de innovación [tesis de maestría, Universidad Nacional de Colombia]. Repositorio Institucional UN. https://repositorio.unal.edu.co/handle/unal/77513

Rubio-Castro, N., \& Luna-García, J. E. (2017). Condiciones laborales psicosociales del personal de la Secretaría Distrital de Salud de Bogotá, 2015. Revista de Investigaciones en Seguridad $\begin{array}{lllll}\text { Social } & y & \text { Salud, } & \text { 34-44. }\end{array}$ http://app.saludcapital.gov.co/revistadigital/Paginas/VerArticulo.aspx

Saravia-Pinilla, M. H. (2005). Desarrollo y evolución de la ergoecología, 1997-2005. Pontificia Universidad Javeriana. Congreso Internacional de Ergonomía Universidad Nacional - Sede $\begin{array}{lllllll}\text { Palmira } & \text { Cali, } & 17, & 18 & \text { y19 } & \text { de }\end{array}$ https://www.researchgate.net/publication/304953546 DESARROLLO Y EVOLUCION D E LA ERGOECOLOGIA 1997-2005

Silva, E. (2011). Revisión documental de la ergonomía en Colombia 1990-2010. Revista Colombiana de Rehabilitación, 10(1), 124-135. https://doi.org/10.30788/RevColReh.v10.n1.2011.83

Stephan, P. F. (2015). Designing 'matters of concern' (Latour): A future design challenge? En W. Jonas, S. Zerwas, K. \& von Anshelm (eds.), Transformation design (pp. 202-226). Birkhäuser Basel, 
Triviño-Cervera, M. A., García-Ubaque, J. C., \& García-Benítez, P. F. (2017). Gestión de la motivación laboral en el personal de enfermería. Revista Salud, Historia y Sanidad, 12(3), 65-76. http://agenf.org/ojs/index.php/shs/article/view/256/247

Velasco-Rey, K. (2016). Análisis de las posturas adoptadas por instrumentadores quirúrgicos $\begin{array}{llll}\text { durante cirugía. Revista Salud Pública, 19(1), } & \text { 112-117. }\end{array}$ http://www.scielo.org.co/pdf/rsap/v19n1/0124-0064-rsap-19-01-00181.pdf

Velásquez-Valencia, J. C., Lange-Morales, K., García-Acosta, G., Gómez-Salazar, L., Luna-García, J. E., Rojas-Fajardo, A. J., Aguilar-Zambrano, J. J. Fandiño-Losada, A., \& Gomes, J. O. (2021). Toward contextual education and research in ergonomics: A Latin American vision. En L. Nancy, N. L. Black, W. P. Neumann \& I. Noy (eds.), Proceedings of the 21st Congress of the International Ergonomics Association (IEA 2021) Volume I: Systems and Macroergonomics. Springer International Publishing. https://www.springerprofessional.de/en/proceedings-of-the-21st-congress-of-theinternational-ergonomics/19122996?

Wilcken, J. (2019). Diseño y manufactura de un mango mejorado para instrumental de cirugía laparoscópica, haciendo énfasis en la ergonomía y la personalización del instrumental [tesis de licenciatura, Universidad de los Andes]. Repositorio UNIANDES. https://repositorio.uniandes.edu.co/bitstream/handle/1992/45654/u827841.pdf?sequence= 1\&isAllowed $=\mathrm{y}$ 